from upper neck pain and/or headache due to bilateral 3rd occipital nerve involvement, excluding other local cervical spine pathologies was confirmed by MRI and previously failed conservative treatment for at least three months prior to enrollment. Sixty adult patients were randomly assigned to one of the two studied groups Group $1(R F, n=30$ ), received bilateral Third occipital nerve Radiofrequency under fluoroscopic guidance or Group 2 (control group, $n=30$ ), received oral prednisolone $10 \mathrm{mg} /$ day. The two groups were then followed-up with neck disability index (NDI), nocturnal neck pain VAS score and headache score every two weeks for three months. Sleep disturbance, sleep disability index were reassessed six months post intervention. Post interventional assessment was done by pain physician who were kept blind to the grouping process.

Results: Neck disability index (1ry outcome), Nocturnal pain VAS, and severity of headache showed significant differences during the whole post-interventional study period. The patients in RF group demonstrated significant improvement of pain in comparison to baseline value over the whole six months with $p$-value < 0.001 as regard to the fore-mentioned three parameters. On the other aspect, the control group patients showed significant improvement in comparison to its baseline value after the 2nd, 12th and 24th weeks only as follows: $(0.001,0.003$, 0.003 for the NDI) ( $p$ values of $0.02,0.01,0.01$ for the nocturnal pain VAS), $(0.001$ $0.009,0.005$ for the headache VAS severity.

Conclusion: Radiofrequency of 3rd occipital nerve is effective in treatment of refractory cervicogenic headache in RA.

Disclosure of Interests: : None declared

DOI: 10.1136/annrheumdis-2020-eular.241

\section{THU0454 SOMATIC SYMPTOMS IN FIBROMYALGIA AND THEIR CORRELATION WITH DRUG TREATMENT}

M. Antivalle ${ }^{1}$, M. Agosti ${ }^{2}$, A. Batticciotto ${ }^{3}$, S. Costi ${ }^{2}$, V. Giorgi ${ }^{1}$, P. Sarzi Puttini ${ }^{1}$.

${ }^{1}$ L. Sacco University Hospital, Rheumatology, Milano, Italy; ${ }^{1}$ L. Sacco University Hospital, Rheumatology, Milano, Italy; ${ }^{3}$ Ospedale di Circolo e Fondazione

Macchi, Rheumatology, Varese, Italy

Background: Drug treatment in fibromyalgia (FM) is often disappointingly ineffective, and there are currently very few data to support therapeutic choices towards a personalized medicine approach.

Objectives: To evaluate the prevalence of selected somatic symptoms in FM, and to study their relationship with drug treatments.

Methods: The study population consisted of 526 patients ( $471 \mathrm{~F} 55 \mathrm{M}$, mean age $47.31 \pm 11.33 \mathrm{yrs}$ ) affected by FM not associated with other rheumatic diseases. All patients were required to compile a questionnaire reporting the presence of 42 somatic symptoms -as suggested (1) - in the last 7 days. Drug usage was assessed by interview.

Results: On average, patients reported the presence of $17.04 \pm 6.68$ symptoms (range 4-35), with ample variations in the prevalence of different symptoms (Fig. 1), ranging from over $95 \%$ (fatigue and muscle pain) to less than $10 \%$, seizures being reported by only 2 patients $(0.4 \%) .31 .1 \%$ of patients were not taking any drug for their FM. The most frequently used drugs were analgesics (ANA, $41.7 \%$ ) followed by benzodiazepines (BD, 29.1\%), SSRIs (16\%), gabapentinoids (GABA, 14,4\%), and NSRI (14.3\%) (Fig. 2). Different drugs were associated with a different spectrum of somatic symptoms: as compared to non users, BD users reported a significantly higher ( $p<0.05$ by chi-square test) prevalence of irritable bowel $(65.4 \%$ vs $52.3 \%)$, fatigue $(98.7 \%$ vs $94.9 \%)$, thinking difficulties ( $78.4 \%$ vs $68.5 \%)$, muscle weakness $(94.1 \%$ vs $81.7 \%)$, abdominal pain $(55.6 \%$ vs $43.9 \%)$, insomnia $(73.9 \%$ vs $56.6 \%)$, depression ( $63.4 \%$ vs $37.2 \%)$, constipation $(60.1 \%$ vs $42.9 \%)$, pain in upper abdomen $(50.3 \%$ vs $40.2 \%)$, nausea ( $53.6 \%$ vs $38.3 \%)$, nervousness $(71.9 \%$ vs $61.5 \%)$, chest pain ( 49.0 vs 37.75$)$, blurred vision (65.4\% vs $53.6 \%)$, dry mouth $(72.5 \%$ vs $52.3 \%)$, itching $(56.2 \%$ vs $44.5 \%)$, vomiting ( $13.7 \%$ vs $7.8 \%$ ), taste change $(22.2 \%$ vs $12.7 \%)$, dry eyes ( $55.6 \%$ vs $41.0 \%$ ), breath shortness $(56.9 \%$ vs $47.7 \%)$, appetite loss ( $33.3 \%$ vs $19.7 \%$ ), painful urination ( $15.0 \%$ vs $8.4 \%$ ), and bladder spasms ( $18.3 \%$ vs $8.6 \%$ ). NRSI users reported a significantly higher prevalence of thinking difficulties, constipation, blurred vision, dry mouth, wheezing, dry eyes, easy bruising. Among GABA users, there was a higher prevalence of thinking difficulties, numbness, insomnia, constipation, nausea, dry mouth, dry eyes, appetite loss, sun sensitivity, easy bruising, and bladder spasms. In no cases a higher prevalence of symptoms was recorded in drug non users vs users.

Conclusion: The usage of different drugs in FM is associated with different somatic symptoms. The higher prevalence of symptoms in drug users as compared to non users raises serious questions concerning the opportunity or the appropriateness of drug selection in FM.
Figure 1. Prevalence of Somatic Symptoms in The Population (\%)
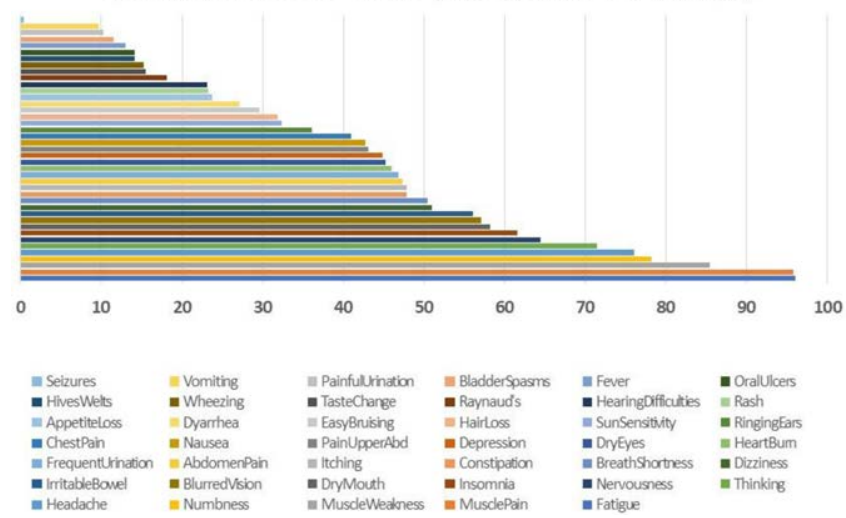

References:

[1] Wolfe F., et al. Arthritis Care Res (Hoboken). 2010 May;62(5):600-10

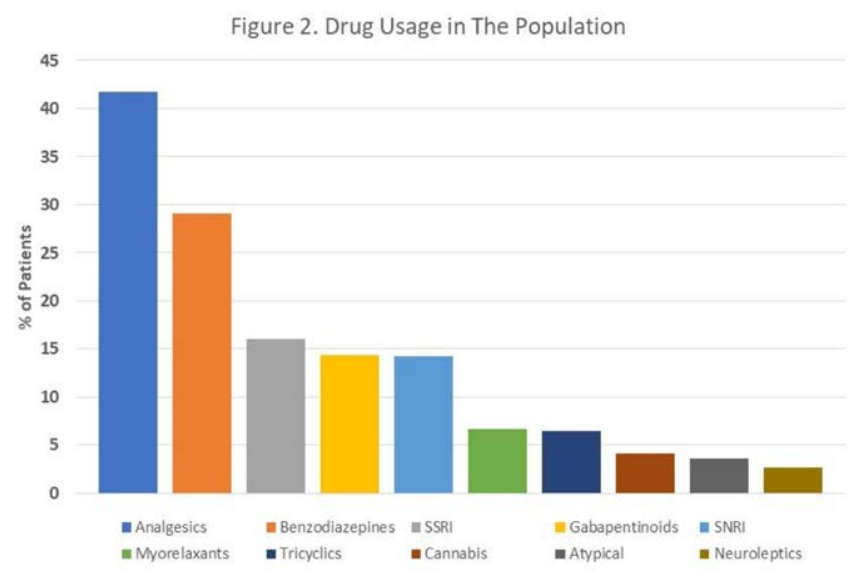

Disclosure of Interests: : None declared DOI: 10.1136/annrheumdis-2020-eular.6427

\section{THU0455 \\ DIFFERENCES IN PSYCHIATRIC COMORBIDITIES AND LIFE ADVERSITIES BETWEEN PATIENTS WITH RHEUMATOID ARTHRITIS ASSOCIATED WITH FIBROMYALGIA AND PATIENTS WITH PRIMARY FIBROMYALGIA}

F. Atzeni ${ }^{1}$, M. Cirillo ${ }^{1}$, I. F. Masala ${ }^{2}$, E. Gerratana ${ }^{1}$, D. Sangari ${ }^{1}$, G. Miceli ${ }^{1}$, P. Sarzi-Puttini ${ }^{3}$, A. Alciati ${ }^{4} .{ }^{1}$ Rheumatology Unit, University of Messina, Messina, Italy; ${ }^{2}$ Trauma and Orthopedic Unit, Santissima Trinità Hospital,, Cagliari, Italy; ${ }^{35}$ Department of Rheumatology, University Hospital ASST Fatebenefratelli Sacco, Milan, Italy; ${ }^{41}$ Department of Clinical Neurosciences, Hermanas Hospitalarias, Villa S. Benedetto Menni Hospital, Albese (Como), Italy ${ }^{2}$ Humanitas Clinical and Research Center, IRCCS, Rozzano, Italy, Como, Italy

Background: Patients with rheumatic arthritis (RA) continue to report significant pain despite apparent disease control by immunosuppressive drugs (1), leading to the hypothesis that central sensitisation (CS) plays a role in the chronic musculo-skeletal pain defining fibromyalgia (FM).

Objectives: The aim of our study was to evaluate the differences in psychiatric comorbidities and life adversities between patients with AR+FM and patients with primary FM (PFM).

Methods: In a observational cross- sectional study patients with PFM and AR+FM were consecutively recruited. The inclusion criteria were an age of 18-70 years; a diagnosis of RA according to the 2010 ACR classification criteria and FM according to the 1990 ACR criteria and 2016 ACR criteria. Lifetime diagnoses of major depression disorder (MDD), panic disorder (PD) and post-traumatic stress disorder (PTSD), three of the most frequently described psychiatric disorders among FM patients, were made with the Structured Clinical Interview for DSM-5. Depressive symptoms were measured using the Zung Self-rating Depression Scale (ZSDS). Childhood trauma was measured using the short form of the Childhood Trauma Questionnaire (CTQ) and stressful events were assessed 
using the Paykel's Interview for Recent Life Events. Pain was assessed using a visual analogue scale (VAS). The Fibromyalgia Impact Questionnaire (FIQ) was also used.

Results: Seventy-seven patients were originally screened, but seven were excluded because of current depressive episode or having a ZSDS of $\geq 60$ or categorized as minimizers of childhood maltreatment at CTQ. The final analysis therefore involved 70 patients, all Caucasians: 30 with PFM and 40 with AR+FM. All patients with PFM and $38(95 \%)$ of the 40 with AR+FM were treated for FM symptoms (antidepressants, pregabalin). The lifetime rates of MDD were significantly higher in PFM vs AR+FM $(76.7 \%$ and $40 \%$ respectively, $p$ $=0.003)$, as well as the rates of PD (50\% and $15 \%$ respectively, $p=0.003)$, whereas there was no difference in PTSD rates. The PFM patients reported significantly higher levels of physical $(p=0.020)$ and sexual abuse $(p=0.011)$ and physical neglect $(p<0.001)$, whereas there was no between-group difference in the levels of emotional abuse $(p=0.912)$ and neglect $(p=0.542)$; consistently, the proportion of sexually abused $(p=0.005)$ or physically neglected patients was also higher in the PFM group $(p=0.023)$. The rates of emotional neglect were high in both groups, without any significant difference between them. The vast majority of $A R+F M$ patients $(90 \%)$ said that only event occurring in the year preceding the onset of FM was RA, whereas the PFM patients mainly reported non-physical events $(36 \%$, particularly the ending of a relationship, or working or financial problems) or no event at all $(40 \%),(p<0.001)$. Binary logistic regression used to identify the factors predicting association of $\mathrm{PFM} / \mathrm{AR}+\mathrm{FM}$ status, showed an association with lifetime major depression, life events preceding the development of FM, and BMI $(p<0.05$ at all).

Conclusion: PFM and SFM differ in psychiatric co-morbidities and environmental adversities, suggesting that the putative common pathogenetic condition of CS may develop through different pathways.

References:

[1] McWilliams DF, Walsh DA. BMC Musculoskelet Disord 2016; 17, 337.

Disclosure of Interests: : None declared

DOI: 10.1136/annrheumdis-2020-eular.3894

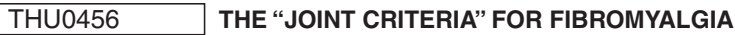 DIAGNOSIS IN RHEUMATOID ARTHRITIS PATIENTS: RELIABILITY COMPARED TO THE 2010 ACR CLASSIFICATION CRITERIA FOR FIBROMYALGIA}

L. J. Ghib ${ }^{1}$, A. Barcic ${ }^{1}$, A. D. Bilous ${ }^{2}$, I. Cozma ${ }^{2}$, M. M. Tamas ${ }^{1,3}$, I. Filipescu $^{1,3}$

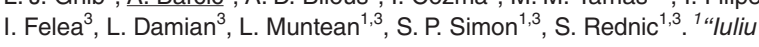
Hatieganu" University of Medicine and Pharmacy, Rheumatology, Cluj-Napoca, Romania; ${ }^{2}$ Rehabilitation Clinical Hospital, Cluj-Napoca, Romania; ${ }^{3}$ Emergency Clinical County Hospital Cluj, Rheumatology, Cluj-Napoca, Romania

Background: A significant proportion of rheumatoid arthritis (RA) patients have concomitant fibromyalgia (FM) (1). Associated FM diagnosis in RA patients can determine worse treatment outcomes compared to patients without FM (1). A difference between tender joint count (TJC) and swollen joint count (SJC) $\geq 7$, also named the "joint criteria" was proposed as being diagnostic for FM in patients with RA. The "joint criteria" were validated against the 1990 ACR Classification Criteria for FM and are easy to apply to patients with RA (2). Since then, the 2010 ACR Classification criteria for FM, which include somatic symptoms besides pain sensitivity, were developed and validated.

Objectives: We aimed to determine the reliability of the joint criteria for fibromyalgia in RA compared to the ACR 2010 Classification Criteria for FM and to compare RA patients diagnosed with FM (FRA) to those without FM in terms of clinical variables.

Methods: We performed a cross-sectional study on RA patients who presented in our department during a 3 months period. Tender joint count (TJC), swollen joint count (SJC), patient global assessment of disease activity (PGA) were determined. DAS28 scores were calculated using CRP. We applied the 2010 ACR Classification Criteria and the joint criteria for FM diagnosis. Kappa agreement coefficient was used to determine the reliability of the joint criteria against the 2010 ACR Classification Criteria for FM in patients with RA. Differences between groups were assessed using Mann-Whitney $U$ test for numerical data or Chi square test for ordinal data.

Results: We included 100 consecutive RA patients, $84 \%$ female, with a mean age of $57.3(12)$ years and mean disease duration of 14(9) years. Twenty-four patients (24\%) had associated FM according to the ACR 2010 Classification Criteria and $22(22 \%)$ patients satisfied the joint criteria for associated FM. The level of agreement between the joint criteria and the ACR 2010 classification criteria for FM was kappa=0.66, $p<0.001$, with a sensitivity of $70 \%$ and a specificity of $93 \%$. FRA patients had similar demographic and disease characteristics compared to RA patients. Patients with FRA according to the joint criteria had significantly higher PGA, DAS28 and $H A Q$ scores, but similar CRP values and SJC compared to RA patients (Table 1).

Table 1 Demographic and clinical data of FRA and RA patients

\begin{tabular}{lccc}
\hline Variable & FRA $\mathbf{n}=\mathbf{2 2}$ & RA $\mathbf{n}=\mathbf{7 8}$ & p-value \\
\hline Age (years) & $60(10.7)$ & $59(12.2)$ & 0.093 \\
Disease Duration (years) & $13.3(13)$ & $12.2(7.5)$ & 0.589 \\
ACPA seropositivity(\%) & 69 & 55 & 0.1 \\
SJC & $2(4)$ & $2(4)$ & 0.7 \\
CRP (g/dl) & $12.8(14.2)$ & $8.1(13.7)$ & 0.06 \\
DAS28CRP & $4(1.7)$ & $3.5(1.2)$ & 0.009 \\
HAQ & $1.75(0.5)$ & $1(0.7)$ & $<0.001$ \\
PGA (mm) & $70(11)$ & $44(23)$ & $<0.001$ \\
\hline
\end{tabular}

Data are expressed as mean (SD) or median (IQR)

FRA- Fibromyalgic Rheumatoid Arthritis; RA- Rheumatoid Arthritis;

ACPA- Anti- citrullinated Protein Antibodies; CRP- C-reactive Protein; SJC- Swollen Joint Count;

DAS28CRP- Disease Activity Score; HAQ- Health Assessment Questionnaire; PGA- Patient Global Assessment

Conclusion: The joint criteria are diagnostic for FM in RA patients with moderate reliability compared to the ACR 2010 Classification criteria. When diagnosed with the joint criteria, FRA patients have higher disease activity scores despite having similar clinical and laboratory inflammatory markers compared to RA patients. References:

[1] Wolfe F, Michaud K. Severe rheumatoid arthritis (RA), worse outcomes, comorbid illness, and sociodemographic disadvantage characterize RA patients with fibromyalgia. J Rheumatol. 2004;31(4):695-700.

[2] Pollard LC, Kingsley GH, Choy EH, Scott DL. Fibromyalgic rheumatoid arthritis and disease assessment. Rheumatology 2010;49(5):924-8.

Disclosure of Interests: : None declared

DOI: 10.1136/annrheumdis-2020-eular.3285

\section{THU0457 LONGITUDINAL ASSOCIATION OF SEDENTARY TIME AND PHYSICAL ACTIVITY WITH SLEEP QUALITY IN WOMEN WITH FIBROMYALGIA: THE AL-ÁNDALUS PROJECT}

M. Borges Cosic ${ }^{1}$, P. Acosta-Manzano ${ }^{2}$, B. Gavilán Carrera ${ }^{2}$, F. EstévezLópez $^{3}$, V. A. Aparicio ${ }^{4}$, V. Segura-Jiménez ${ }^{5}$, M. Delgado-Fernández ${ }^{2}$. ${ }^{1}$ University of Granada, Faculty of Sport Sciences, Department of Physical Education and Sport, Granada, Spain; ${ }^{1}$ University of Granada, Faculty of Sport Sciences, Department of Physical Education and Sport, Granada, Spain; ${ }^{3}$ Erasmus MC University Medical Center, Department of Child and Adolescent Psychiatry/Psychology, Rotterdam, Netherlands; ${ }^{4}$ University of Granada, Faculty of Pharmacy, Department of Physiology, Granada, Spain; ${ }^{5}$ University of Cádiz, Faculty of Physical Education, Department of Physical Education, Cádiz, Spain

Background: Sleep disturbances are common in fibromyalgia, and influences quality of life. Recent literature has suggested that non-pharmacological treatments (e.g., physical exercise and cognitive behavioural therapy) may help to improve sleep quality (SQ) and the management of fibromyalgia ${ }^{1}$. In this regard sedentary time (ST) and physical activity (PA) intensity levels could play a role on $S Q$ in this population ${ }^{2}$. However, evidence is scarce and mainly based on cross-sectional data.

Objectives: This study aimed to examine the longitudinal associations (2and 5-year follow-up) of ST and PA intensity levels with SQ in women with fibromyalgia.

Methods: In this prospective cohort study, women diagnosed with fibromyalgia (age: $51.4 \pm 7.6$ years) with completed data were included at baseline $(n=409)$, at 2-year follow-up $(n=214)$ and at 5-year follow-up $(n=218)$. Sedentary time and PA intensity levels (light and moderate-to-vigorous [MVPA]) were assessed using triaxial accelerometers worn for consecutive 7 days. The percentage of time spent in different behaviours was calculated (e.g., (ST/accelerometer wear time) $\times 100$ ). The SQ global score was calculated as a sum of all components (score 